\title{
Morfología del verbo garífuna ${ }^{1}$
}

\author{
J. Diego Quesada \\ Universidad Nacional, Costa Rica
}

\begin{abstract}
resumen
Presenta una descripción de la morfología del verbo garífuna, única lengua arahuaca hablada en Centroamérica, desde una perspectiva funcionaltipológica. Es un avance respecto a estudios previos por tratar la dimensión verbal como una unidad temática. Se ofrece un inventario de las formas verbales del garífuna, haciendo referencia a la formación de verbos, a las clases verbales, a la existencia de formas cortas, a la distinción entre verboides y verbos plenos, así como a las formas que cumplen el perfil de auxiliares, y un recuento de las categorías verbales del garífuna.
\end{abstract}

\begin{abstract}
A detailed description is given here of Garifuna, the only Arawakan language spoken in Central America, regarding its verb morphology from a typological-functional perspective. It provides a more encompassing view in comparison to previous studies insofar as it describes the verbal dimension as a thematic unit. It provides an inventory of Garifuna verb forms, with special emphasis on verb-formation, verb classes, the existence and distribution of short forms, as well as the distinction between verboids (nominalized forms) and full verbs; additionally, reference is made to forms fulfilling the profile of auxiliaries, and the paper next identifies all relevant grammatical categories of the Garifuna verb.
\end{abstract}

1 Versión revisada de la ponencia leída en el marco del I Simposio Internacional del Programa de Lingüística Centroamericana (PROLINCA) «Centroamérica: un microcosmo lingüístico, llevado a cabo los 7 y 8 de mayo 2012, en la ciudad de Heredia, en el campus Omar Dengo de la Universidad Nacional de Costa Rica. Recibido: 29 de enero de 2012; aceptado: 1 de abril de 2012.

2 Escuela de Literatura y Ciencias del Lenguaje. Correo electrónico: jquesad@una.cr 
Palabras clave: garífuna, lenguas arahuacas, lenguas centroamericanas Keywords: Garifuna, Arawakan languages, languages of Central America

\section{Introducción}

Este trabajo ofrece una descripción detallada de la morfología del verbo garífuna, única lengua arahuaca hablada en Centroamérica, desde una perspectiva funcional-tipológica. Aunque no es la primera descripción del verbo garífuna, pues la primera es de Breton ${ }^{3}$, así como varios estudios de Taylor, sintetizados en Taylor ${ }^{4}$, y otros menos especializados $\left(\right.$ Suazo $^{5}$; Devonish y Castillo $\left.{ }^{6}\right)$, representa un avance respecto de las anteriores, en tanto que trata la dimensión verbal como una unidad temática, mientras que las otras tocan temas verbales específicos. Ello permite revisar y corregir afirmaciones sobre diversos aspectos del verbo garífuna existentes en la escasa literatura sobre el tema. Un trabajo muy difundido en Honduras es el de Suazo (1991), concebido como una guía para aprender la lengua, matizado por un intento por sistematizar algunos aspectos de la lengua, tanto en lo nominal como en lo verbal. En este último aspecto, el estudio queda debiendo, pues presenta tres deficiencias importantes: 1) los paradigmas verbales son presentados

3 Raymond Breton, Grammaire caraïbe (Auxerre: Gilles Bouquet, imprimeur ordinaire du ROY, 1667); puede consultarse una versión electrónica: <http://www.archive.org/stream/ grammairecaraib00adamgoog\#page/n48/mode/1up>. También de Breton, Dictionnaire françois-caraïbe (Auxerre: Gilles Bouquet, imprimeur ordinaire du ROY, 1666; versión electrónica: <http://www.archive.org/stream/dictionairefran00platgoog\#page/n6/mode/1 up>.

4 Douglas R. Taylor, Languages of the West Indies (Baltimore: Johns Hopkins Press, 1977). Otros estudios de Taylor: «Lexical borrowing in Island-Carib», Romance Philology 16 (1962): 143-52: «Island-Carib, IV; Syntactic notes, texts», International Journal of American Linguistics 24 (1) (1958a.): 36-60; «Carib, Caliban, Cannibal», International Journal of American Linguistics 24.2 (1958b.): 156-7; «Island-Carib, II: Word classes, affixes, verbs, nouns», International Journal of American Linguistics 22.1 (1956a.): 1-44; «Island-Carib, III: Locators, particles», International Journal of American Linguistics 22.2 (1956b.): 138-50; «Phonemes of the Hopkins (British Honduras) dialect of Island-Carib», International Journal of American Linguistics 21.2 (1955): 233-4; The Black Carib of British Honduras (Nueva York: Wenner-Gren Foundation, 1951).

5 Salvador Suazo, Conversemos en garífuna, 2a ed. (Tegucigalpa: Editorial Guaymuras, 1991).

6 HubertDevonishyEnitaCastillo, «SomeFeaturesoftheMorpho-SyntaxofSimpleSentencesinGarifuna».Ponencia presentadaante The University of the WestIndies, Schoolfor Continuing Studies, Belize Country Conference, 2001. Puede consultarse también la versión electrónica: <http://ebookbrowse. com/some-features-of-the-morpho-syntax-of-simple-sentences-in-garifuna-doc-d62397164>. 
siguiendo el esquema del español (al igual que lo hizo Breton con el francés 300 años antes), y en algunos casos con nomenclatura un tanto sui generis; 2) las conjugaciones»de los verbos solo aparecen en construcciones sin objeto, lo cual en el caso del garífuna es trágico, pues la presencia de objeto es una de las variables que determinan la distribución de los paradigmas; 3) la descripción es bastante superficial e incompleta ${ }^{7}$. Este trabajo consta de dos secciones principales; en el segundo apartado se presenta un inventario de las formas verbales del garífuna; se hace referencia a la formación de verbos, a las clases verbales, a la existencia de formas cortas, a la distinción entre verboides y verbos plenos, así como a las formas que cumplen el perfil de auxiliares. En el tercer apartado se hace un recuento de las categorías verbales del garífuna, donde se destaca la de aspecto como la principal y la de tiempo como la más marginal. Finalmente, se presentan las conclusiones del estudio. Los datos se extraen principalmente del trabajo de campo realizado en las comunidades aledañas a Trujillo, en el departamento de Colón, Honduras, y forman parte del proyecto de elaboración de la gramática del garífuna en el marco del Programa de Lingüística Centroamericana de la Universidad Nacional ${ }^{8}$.

\section{Formas verbales}

\section{Clases verbales y formación de verbos}

Los verbos en garífuna se reconocen por su terminación en $-a$, en lo que podría denominarse su forma infinitiva ${ }^{9}$. No obstante, según de la secuencia de segmentos que preceden al marcador de

7 llustrativo de este proceder es una fórmula utilizada con bastante frecuencia por el autor a la hora de presentar las diferentes estructuras: «para formar el tiempo $\mathrm{X}$ basta utilizar este esquema» y seguidamente presenta un paradigma; pero no ahonda en las propiedades distribucionales del mismo.

8 El autor agradece a Balbina Chimilo, Simeón Marín y Nolvia Loredo, todos hablantes nativos del garífuna de Colón (Honduras), por su generosa colaboración durante las arduas sesiones de trabajo en sus comunidades.

9 Hay unos pocos verbos producto de préstamos, los cuales no se pertenecen a esas clases por terminar en -ü, como operarü 'operar', konektarü 'conectar', multiplikarü 'multiplicar'; esa terminación, sin embargo, no tiene implicaciones para su comportamiento morfosintáctico. 
infinitivo, es posible distinguir aparentes clases. Para este estudio se cuantificaron todas las entradas correspondientes a verbos, contenidas en la primera parte, garífuna-español, de la obra de Sabio y Ordóñez ${ }^{10}$ y se obtuvo un total de 1429 verbos, distribuidos en doce clases: - $h a,-d a,-r a,-g u a,-c h a,-g a,-n a,-y a,-b a,-w a,-l a, \mathrm{y}$ $-m a$; sin embargo, no todas esas clases son productivas; tampoco tienen implicaciones en la morfosintaxis de la lengua, pues en muchos casos sus presuntos significados originales se han perdido. Como se muestra en el cuadro 1 de Sabio y Ordóñez, las primeras cuatro clases representan el $91 \%$ del total de los verbos, el remanente $9 \%$ queda repartido entre las restantes ocho clases; y de esas ocho clases, -cha incluye el $6 \%$ y $-g a$ el $2 \%$, es decir, el $8 \%$, mientras que seis clases —o sea la mitad - se reparten el sobrante $1 \%$.

\section{Cuadro 1. Clases verbales: cantidad y porcentajes}

\begin{tabular}{|c|c|c|c|c|c|c|c|c|c|c|c|c|}
\hline$-\boldsymbol{h a}$ & $-\boldsymbol{d a}$ & $-\boldsymbol{r a}$ & $\boldsymbol{- g u a}$ & $-\boldsymbol{c h a}$ & $\boldsymbol{- g} \boldsymbol{a}$ & $-\boldsymbol{n a}$ & $-\boldsymbol{y a}$ & $\boldsymbol{- b a}$ & $-\boldsymbol{w a}$ & $-\boldsymbol{l a}$ & $-\boldsymbol{m a}$ & Total \\
\hline 470 & 309 & 259 & 252 & 85 & 22 & 4 & 3 & 5 & 17 & 2 & 1 & 1429 \\
\hline 33 & 22 & 18 & 18 & 6 & 2 & 0.28 & 0.21 & 0.35 & 1.19 & 0.14 & 0.07 & $100 \%$ \\
\hline
\end{tabular}

Las clases mayoritarias se reconocen por sufijos que originalmente - y que aun en la actualidad, en muchos casos, su significado no es totalmente opaco- expresaban distinciones aspectuales, entre ellas atelicidad $(-h a)$, incoatividad $(-d a)$, puntualidad o telicidad $(-r a)$, y diátesis media $(-g u a)$. Ejemplos de ello son raíces como abini- 'bendecir' o abürü- 'escribir' que con los sufijos -ha y -da expresan situaciones atélicas e incoativas, respectivamente. Asimismo existen pares atélicos $(-h a)$ y télicos $(-r a)$ como son achagaha 'botar algo varias veces' vs. achagara 'botar una cosa o varias una

10 Aunque Suazo (2011) contiene considerablemente más entradas, se utilizó a Sabio y Ordóñez (2006) porque la segunda especifica los verbos que tienen formas cortas, mientras que la primera no. Como se ve en la siguiente subsección, ese es un parámetro relevante en la descripción de la morfología de esta lengua. Salvador Suazo. Lila Garifuna. Diccionario Garífuna-Garífuna-Español (Tegucigalpa: Litografía López, 2011). Fernando Sabio y Celia Ordóñez, Hererun Wagüchagu (La Ceiba: Asociación Misionera Garífuna, 2006). 
vez'; la raíz abadisei- 'bautizar' puede tomar el sufijo télico (abadiseira), el atélico (abadiseiha) y el medio (abadiseirúa), este último con una variante de - gua. Aunque existen formas que podrían considerarse media tantum, como adumuridagua 'reunirse' o ahuduragua 'torcerse' e inchoativa tatum como abinaduda 'envejecer' o abufida 'hincharse el cuerpo', entre otras, pues no tienen contrapartes télicas ni atélicas, esta caracterización no es exhaustiva, pues existen formas no terminadas en - gua y $-d a$ que expresan situaciones medias e incoativas, respectivamente; tal es el caso de abuluha 'sumergirse' y asurusuha 'desnudarse'.

En muchos otros casos, los sufijos han ido perdiendo las distinciones originales ${ }^{11}$. Por ejemplo, existen casos en que el aparente sufijo medio - gua ha perdido totalmente ese rasgo, como en $a b a-$ racha que significa 'pringarse', mientras que abarachagua significa 'pringar a otro'. Es habitual que los objetos de formas verbales terminadas en -gua tiendan a ser colectivos, plurales. Evidencia de la opacidad del sufijo medio es la tendencia a reforzar situaciones medias con el pronombre reflexivo, como en achuinra-gua ungua 'machucarse'. La creciente opacidad se manifiesta también en la adquisición de significados no relacionados, como ocurre en casos como ásuha 'variar, dudar, estar indeciso' vs. ásura 'terminar', donde la oposición no es de telicidad sino que es ya lexemática. Así pues, es lícito concluir que las clases verbales del garífuna son principalmente cuatro y que los sufijos que las distinguen están en proceso de creciente opacidad; de igual manera, estas clases son esencialmente semánticas, pues no dan origen a paradigmas morfosintácticos como ocurre en las lenguas romances, por ejemplo.

Hay indicios que permiten postular la clase en $-h a$ como la básica o no marcada. En primer lugar, constituye la clase con mayor número de miembros; una tercera parte $(33 \%)$ de los verbos de la

11 Taylor (47) interpreta la creciente opacidad y lexicalización de los sufijos verbales en términos de formas productivas y no productivas e incluye a tres de las cuatro principales (no incluye la clase incoativa) entre las productivas. 
muestra pertenece a esta clase. Segundo, la gran mayoría de neologismos vienen a caer en esta clase; a partir de préstamos se crea una raíz a la que se le aplica el proceso de parasíntesis, prefijando $a$ $e$ - y sufijando $-h a$, especialmente meter $\rightarrow$ e-mete-ha 'entrometerse', sumar $\rightarrow$ a-suma-ha, sal $\rightarrow$ a-salu-ha; ingl. box $\rightarrow$ a-baksi-ha 'boxear'; fr. roi 'rey' $\rightarrow$ a-rue-ha 'reinar'. Tercero, ante la existencia de formas terminadas en otros marcadores de clase (p.ej. -ra y $-d a$ : emeteha alterna con emetera, uno atélico y el otro télico; o abisida que alterna con abisidaha, uno incoativo y el otro atélico), la forma lemática, o de citación, tiende a ser la terminada en $-h a^{12}$. Cuarto, la clase en -ha es la clase con más miembros, pero con menos formas cortas (solo un $18 \%$ ), lo que puede interpretarse como característico de su estatus no marcado o básico.

\section{Formas cortas}

Una característica de los verbos garífunas es la existencia de formas cortas que alternan con las largas, sobre todo en cuanto a la distinción entre formas plenas y formas nominalizadas (ver las siguiente sección); las formas cortas se utilizan, cuando el verbo las posee, en la expresión de categorías verbales como el aspecto (excepto el progresivo) y el futuro sintético, así como en la construcción objetiva. De la muestra analizada, es decir, de los 1429, el $38.20 \%$ (546 verbos), posee una forma corta, tal y como se aprecia en el cuadro 2.

12 Cierto es también que en muchos casos de verbos que tienen forma larga y forma corta (ver explicación respectiva), la segunda se utiliza como forma de citación. Ahora bien, la gran mayoría de los verbos en - $h a$ no tienen forma corta, lo que da como consecuencia que sean formas de esa clase las utilizadas como lemas. También es cierto que en algunos casos la forma básica no es la terminada en $-h a$ por determinación semántica; se trata principalmente de aquellos casos en que $-h a$ expresa iteratividad antes que una situación atélica; así pues, abadiseira 'bautizar' (télico), aparece como forma lemática antes que abadiseiha 'bautizar varias veces' (atélico); lo mismo ocurre con amura 'defecar' vs. amuraha 'defecar repetidamente'; en ambos casos la realidad extralingüística no marcada es la forma puntual, no la iterativa. 


\section{Cuadro 2. Porcentaje de formas cortas de los verbos garífunas}

\begin{tabular}{|l|c|c|}
\hline$-h a$ & 86 & $6.01 \%$ \\
\hline$-d a$ & 154 & $10.77 \%$ \\
\hline$-r a$ & 143 & $10.00 \%$ \\
\hline$-g u a$ & 108 & $7.55 \%$ \\
\hline$-c h a$ & 37 & $2.58 \%$ \\
\hline$-g a$ & 9 & $0.62 \%$ \\
\hline$-n a$ & 2 & $0.13 \%$ \\
\hline$-y a$ & 0 & $0.00 \%$ \\
\hline$-b a$ & 3 & $0.20 \%$ \\
\hline$-w a$ & 1 & $0.06 \%$ \\
\hline$-l a$ & 2 & $0.13 \%$ \\
\hline$-m a$ & 1 & $0.06 \%$ \\
\hline Total & $\mathbf{5 4 6}$ & $\mathbf{3 8 . 2 0} \%$ \\
\hline
\end{tabular}

Sobre las formas cortas de los verbos del garífuna hay que hacer algunas observaciones. Existen casos en los que la forma corta es igual para dos verbos con terminaciones diferentes pero ya lexicalizadas, como agubuda 'pudrir' y agubuha 'provocar'; en ambas es gubú. En otros, como emenigiralemenigida 'confiar, tener esperanza', ambas formas aparecen como meros sinónimos con idéntica forma corta, hemenigi; es decir, los sufijos se han desemantizado; de igual forma, abayuha y abayuragua 'arrebatar' tienen la misma forma corta: bayú; abeiha 'chapear' y abeicha 'castigar', poseen una sola forma corta, bei. En otros casos la terminación tampoco hace diferencia pero solo una de las dos formas largas tiene forma corta; es el caso de abiniha y abinira 'bendecir', pero solo la segunda tiene forma corta: biní. El caso contrario también se da; a saber, en los que la terminación hace diferencia y ambas toman la misma forma, como ocurre con abouncha 'batir agua al nadar' vs. abounha 'reventar de las olas'; ambas tienen la misma forma corta, boun. 
Las formas cortas son en muchos casos predecibles, formándose mediante la elisión de la primera sílaba y del sufijo de clase; en el caso de la clase media el sufijo - gua casi siempre aparece en las respectivas formas cortas. Algunos ejemplos de formas cortas por claseson:

Clase-ha:

abaküha 'defecar'

efeindiha 'pintar'

éfereha 'desgranar'

esefuha 'salvar a varios en general

ewerediha 'advertir' bakü

feindi

feré

sefu

wéridi

Clase-da:

añuluda 'suavizar, aliviar'

ñulú

asilaküda 'destemplar un mecate'

silakü

asiriseda 'oler a orines'

sirise

asusereda 'suceder'

susere

ásüda 'calentarse'

sü

Clase -ra:

áchara 'esconder'

cha

acharara 'equivocarse'

chara

ácharura 'clavar, punzar'

charú

achawara 'atraer, jalar todo de un solo'

chawá

achokura 'estrangular'

choku

\section{Clase-gua:}

achalaragua 'enjuagar'

chalaguaü

achamuragua 'lanzar'

chamugua

afaragadagua 'desenvolver (mecate)'

fararagua

ínsiñedagua 'amarse'

hínsiñegua

ímiragua 'puyar, traspasar'

miragua

En otros casos, las formas cortas son supletivas, como en las siguientes: 
ata 'beber'

íchagua 'dar, poner'

anüga 'traer, llevar'

eibuga 'caminar'

eiga 'comer'

íchiga 'dar' gurá

rugua

barï

ñ̈̈dün, -idin, -oudin

hou

$r u$

\section{Formas nominalizadas y verbos plenos}

Una característica del sistema verbal del garífuna la constituye la existencia de dos grupos principales de formas verbales, que se denominarán verboides y verbos plenos, respectivamente. Los primeros son formas caracterizadas por la indexación del sujeto prefijado - y objeto - sufijado - pero total ausencia de marca aspectual (por ejemplo, b-ariha-na $2 \mathrm{sG}$-ver-1 $\mathrm{sG}$ 'tu verme', 'me ves'); en el caso de las construcciones intransitivas, solamente aparece el sujeto prefijado (p. ej. n-arumuga $1 \mathrm{sG}$-dormir 'mi dormir', 'yo duermo', 'voy a dormir'). Asimismo los verboides pueden portar un sufijo nominalizador (p. ej. b-arihi-ni-na $2 \mathrm{sG}$-ver-nom-1SG 'tu verme', 'me ves', transitivo, y b-ereder-un 2 sG-quedar-nom 'tu quedarte', 'te quedás', intransitivo $)^{13}$. Esa característica las asemeja a construcciones nominales posesivas, en las que el sufijo de persona expresa al poseedor. La naturaleza nominal de los verboides se manifiesta en el cambio de la vocal verbal $-a$ a $-u$ (y a $-i$ en otros verbos), lo cual según Taylor ${ }^{14}$ indica nominalización de la forma verbal en cuestión. Taylor denomina a estas formas indefinidas o no aspectuales y de ellas dice que adquieren su delimitación témporo-aspectual dependiendo del contexto en el que aparecen, lo cual es correcto hasta

13 En garífuna los sustantivos presentan dos formas, una forma poseída, utilizada como forma lemática de sustantivos inalienables, y una no poseída, utilizada como forma lemática de sustantivos alienables. Existen varios sufijos de posesión, entre ellos $-V n$, que parece aplicarse solo a sustantivos alienables, en principio independiente de la alienabilidad de los sustantivos, $\mathrm{y}-n i$.

14 Taylor, 50. 
cierto punto ${ }^{15}$. No obstante, Taylor no menciona dos características, de naturaleza sintáctica, propias de los verboides. La primera es que son formas casi — con pocas excepciones - exclusivamente subordinadas; es decir su locus sintáctico es la oración subordinada, como se ilustra en (1).

\section{(1) Sagü t-afurid-un t-adunrag-un ha-mat}

Cada 3sG.f-salir-nom 3sG.f-encontrase-nom 3pl-con

-agani-gu ha-ma l-umari-gu lun h-ageindag-un 3sG.f-enemiga-pl 3pl-con 3sG.f-esposa-pl para 3pl-pelear-nom dari lun l-anüg-un-i Bungiu

hasta que 3sG.m-llevar-nom-3sG.m Dios

'Cada vez que salía [lit. 'cada salida de ella'] se encontraba con sus enemigas, las esposas de él, para pelearse hasta que Dios se lo llevó'

La segunda característica sintáctica de los verboides tiene que ver con un principio sintáctico, según el cual la posición inicial de cláusula está reservada a operadores sintácticos, entre ellos subordinadores, adverbios, pronombres personales libres y verbos plenos. Así pues una oración puede iniciar como en (2a), pero no como en (2b); lo contrario también es posible (2c-d); es decir, puede iniciar como en (2c), pero no como en (2d). Con objetos directos funciona de igual manera; para decir 'El hombre abrió la puerta', se debe iniciar con una forma plena (3a), una construcción de foco (3b) o con un adverbio en posición inicial (3c), pero no con un verboide (3d), excepto que el sujeto sea definido (3e). Nótese que sintácticamente $(3 b-c)$ no son oraciones subordinadas propiamente, sino que lo que

15 Por ejemplo, dependiendo de la presencia del sufijo nominalizador las formas tienden a tener una lectura témporo-aspectual específica; considérense las siguientes tres formas, todas sin marca aspectual: $n$-atatira-li, $n$-atatira-i y $n$-atatir-un-i (< atatira 'comenzar'); los hablantes consecuentemente las traducen como 'lo voy a comenzar', 'lo comienzo' y 'lo comencé', respectivamente. 
determina el uso de la forma nominalizada es la imposibilidad de aparecer en posición inicial (más el estatus definido del sujeto) ${ }^{16}$.
(2a) Arienga-ti-na
l-un
Karlos
lun...
Decir-aor-1sG 3sG.m-dat
Carlos
que...
'Le dije a Carlos que...'
(2b)
*n-arieng-un l-un
1sG-decir-nom 3sG.m-dat
Karlos
lun...;
'Le dije a Carlos que...'
(2c) Dan le n-arieng-un
Cuando 1sG-decir-nom
Carlos que
'Cuando le dije a Carlos que...'
(2d) *
*Dan le arienga-ti-na
Cuando decir-aor-1 $\mathrm{sG}$
l-un
3sG.m-dat
'Cuando le dije a Carlos que...'
(3a) Dara-t- $i$
eyeri
Abrir-aor-3sG.m hombre
l-un
3sG.m-dat
le
bena
dem.m puerta
Karlos lun...
Carlos que
Karlos lun....
Carlos que...

'Ese hombre abrió la puerta'

(3b)Eyeri le l-adar-un

bena

Hombre dem.m 3sG.m-abrir-nom

'eSe hombre abrió la puerta [Fue ese hombre que abrió la puerta]'

(3c)

Aban l-adar-un

Entonces 3SG.m-abrir-nom eyeri

hombre le bena

dem.m puerta

\section{'Entonces el hombre abrió la puerta'}

16 Las formas nominalizadas guardan una similitud con el conocido infinitivo personalizado del portugués: O professor deu um prazo de cinco dias para os alunos estudarem bastante para a prova, o en $O$ bom é sempre lembrarmos desta regra, y casos similares en español, como Para poder yo $\sim$ yo poder ayudarle necesito más detalles, o Al Pedro saber la verdad, se quitó la vida. En los tres casos, garífuna, español y portugués se trata de formas nominalizadas; la diferencia entre el garífuna y las dos lenguas romances radica en que en las últimas no existe la restricción sintáctica apuntada arriba, ni la posibilidad de aparecer en una construcción de foco (cfr. 58b), por ejemplo en oraciones escindidas: *Fue Pedro (que) saber la verdad o *Es yo que poder ayudarle. Nótese que en el equivalente garífuna la forma nominalizada está fungiendo como oración principal. Tampoco pueden aparecen seguidos de una conjunción: *Entonces Pedro saber la verdad. No obstante lo anterior, la función de los verboides es en términos generales comparable con la de las formas nominales de los verbos en lenguas indoeuropeas. 

(3d) *l-adar-un
bena
3sG.m-abrir-nom
puerta
'Ese hombre abrió la puerta'

$\begin{array}{lll}\text { (3e) } l \text {-adar-un } & \text { eyeri } & \text { le bena } \\ \text { 3sG.m-abrir-nom } & \text { hombre } & \text { dem.m puerta } \\ \text { 'Ese hombre abrió la puerta' }{ }^{17} & \end{array}$

Los verbos plenos no solo son portadores de todas las categorías verbales, sino que no tienen las restricciones distribucionales de los verboides, salvo, claro está aparecer en los contextos de los verboides. Dan origen a dos tipos de formas verbales, simples y compuestas; las segundas se construyen sobre la base de auxiliares (ver la siguiente sección) y su uso está determinado por la indexación del objeto directo en la cadena verbal y del estatus del objeto como definido, individuado. La estructura morfológica de un verbo pleno simple se resume en (4).

(4) Forma plena: $[$ suj- $\mathbf{V}-\mathbf{a k t}-\mathbf{t a m}-\mathbf{o b j}]$
Forma corta: $[\mathbf{V - t a m - a k t - S u j ]}$

Según se desprende de (4), en las formas plenas cortas no se indexa el objeto; éste tiende a aparecer como un sintagma nominal pleno. Para indexar a ambos participantes, las formas cortas $-\mathrm{y}$ también las plenas en las algunas dimensiones aspectuales- requieren de un auxiliar. Éstos constituyen materia de la siguiente subsección. El espacio reservado para sufijos de modo de acción o aktionsarten, akt en (4), lo ocupan no solo los sufijos de marcas verbales cuando no están lexicalizados (por ejemplo, el medio -gua, el atélico - ha -llamado «tensivo» por Taylor) ${ }^{18}$, sino también el causativo -güda,

17 Los sustantivos sin marca pueden tener una lectura tanto definida como indefinida. En estos ejemplos lo que se demuestra es la agramaticalidad de la construcción causada por la ausencia de un sintagma nominal sujeto explícito.

18 Taylor, 48. El orden de los morfemas está determinado por el grado de lexicalización de estos sufijos; así pues, los sufijos de aktionsart, cuando están lexicalizadas, anteceden al sufijo causativo 
el pasivo - $(o) w a$, el restrictivo -rügü, el restrictivo negativo $-d i$ 'no solo' o el iterativo -ya. Algunos de estos sufijos de aktionsart aparecen en los verboides también, como lo hiciera notar Taylor.

\section{Auxiliares y construcciones compuestas}

Tanto Taylor como Devonish y Castillo ${ }^{19}$, concuerdan que existen formas que cumplen el perfil de auxiliares, aunque no necesariamente en sus funciones, ni en su cantidad. Taylor identifica un total de seis auxiliares: $u b a$, al que define como un marcador de modo irrealis con tres funciones principales, futuro, imperfecto y relativizador; $(i)$ ña, para expresar aspecto progresivo; uma para expresar aspecto perfectivo; ani (producto de la unión de (um)a y el nominalizador -ni) para producir participios; iabu para expresar intencionalidad, y el condicional hamuga. De esos seis, Devonish y Castillo solo identifican tres: uba para expresar futuro, uma para expresar aspecto perfectivo y umut para expresar situaciones puntuales. En la variedad estudiada (Trujillo y alrededores), existen todas estas formas, pero con ligeras diferencias morfológicas y funcionales. Por ejemplo, los marcadores de futuro y progresivo han alcanzado un grado de morfologización que prácticamente ya funcionan como sufijos; por ejemplo, la construcción que Taylor ${ }^{20}$ brinda como forma de futuro ${ }_{\mathrm{VP}}\left[{ }_{\mathrm{V}}[a r i h a]_{\mathrm{V} \text { Aux }}[n-u b a-d i-b u]_{\mathrm{Aux}}\right]_{\mathrm{VP}}$ 'te veré' no se utiliza, siendo la que el mismo autor denomina como «alternativa», la que los hablantes dicen conocer, a saber ${ }_{\mathrm{V}}[n \text {-arihu-ba-di-bu}]_{\mathrm{V}}$. La forma de Trujillo y alrededores revela un grado mayor de cohesión y morfologización, en tanto que el prefijo de persona pasa del auxiliar a la raíz, dejando a éste en estado de afijo. También es prácticamente

(-güda), pero cuando no lo están lo siguen; por ejemplo, en el verbo ibagua 'cortar', Ta secuencia -gua está lexicalizada; al aplicársele el sufijo causativo, éste aparece al final de la cadena: ibaguagüda 'hacer cortar', pero en casos como el verbo abadiseiha 'bautizar varias veces', donde el sufijo

-ha expresa la atelicidad, el sufijo causativo lo precede abadiseira-güda-ha 'hacer que se bautice varias veces'.

19 Taylor; Devonish y Castillo.

20 Taylor, 52. 
sufijo en preguntas como (5), donde no es posible analizar al pronombre interrogativo como un prefijo.

¿Ka-ba b-adüga haruga?
Qué-fut 2sG-hacer mañana
¿Qué vas a hacer mañana?’

Hay que detenerse en los usos de este auxiliar. Como ha quedado dicho, Taylor lo analiza como un marcador de modo irrealis, con tres funciones; una de ellas es futuro, ya comentado, y las otras dos las denomina funciones relativas, de objeto y sujeto, del aspecto imperfectivo. La primera se hace aparente en (6); en esa construcción, según Taylor ${ }^{21}$ «only a suffixed marker of third person can occur, and this refers and attaches the verb to a foregoing antecedent with which it agrees in gender or plural number». El otro uso de $-b a$ aparece en las construcciones que se pueden denominar de foco, como en (7). A esa función Taylor le denomina «relativa objetiva» porque «relates the following personal suffix to the rest of the verb as its goal». En ambos casos, la función de $-b a$ es evidentemente la de modificar a un referente nominal; por ello, esa función en este estudio se denominará relativizadora. Un caso más claro de marcador de aspecto imperfectivo de $-b a$ se ofrece en $(8)^{22}$.

(6)
¿Ka $\quad b$-adagu-ba-i?
Qué 2sG-hacer-rel-3sG.m
¿¿Qué hacías? [lit. ‘¿Qué es lo que hacías?’]

21 Taylor, 50 .

22 Devonish y Castillo analizan el cambio de referencia témporo-aspectual (futuro e imperfectivo) de la siguiente manera:

The likely analysis here is that sentence initial pronouns such as $k a$ and ligiya are potential bearers of the - ba- marker. If they are not so marked, the sentence is interpreted as having present rather than future reference. When $-b a$ - appears on the following verbal stem, the time reference of the sentence is already established as the present and the meaning of 'before, prior' produces a past meaning, i.e., a meaning of 'prior to the present'. A diferencia de esos autores, los usos de $-b a$ como en (6) aquí se analizan como casos de relativización. Pero para los casos de imperfectivo como (8), su análisis sí se aplica. 

(7) Ligia
afar-uba-li
animalu
3SG
matar-rel-3sG.m
animal
'Él fue quien mató al animal'
(8) L-umari agumadir-uba-li
3sG.m gobernar-ipfv-3sG.m
'La mujer lo manda'

Casos más claros de auxiliar, concebido como portador de morfemas (como do en inglés, o haber en español), lo constituye (9), donde sigue a la forma negativa del verbo igera 'dejar'. En (9) también aparece la forma lubei (ostensiblemente $l-u b a-i$, 3sG.m-uba3sG.m), la cual ya es una especie de estativo invariable -de ahí que las fuentes como Sabio y Ordóñez ${ }^{23}$ o Cayetano ${ }^{24}$ ya no la escriban como <lubai>-; esa función se hace clara en la definición de source 'fuente' dada por Cayetano ${ }^{25}$, reproducida aquí como (10). Evidentemente, las diversas formas y funciones de $u b a$ analizadas aquí representan grados diversos de gramaticalización del auxiliar ${ }^{26}$.
(9)
Ligia m-igir-un
$\eta-u b a-i$
lubei $\quad b$-un
Por eso neG-dejar-nom
1sG-fut-3 SG-m estar
2SG-dat
'Por eso no te lo voy a dejar'
(10) le
ñéi-gie-lubei
l-iabi
aban katei
dem.m
ahí-donde-estar
3sG.m-venir
uno cosa
'El lugar de donde viene una cosa'

Una situación similar ocurre con el marcador de aspec-

to progresivo, el cual para expresar esa dimensión aspectual es un

\footnotetext{
23 Sabio y Ordóñez.

24 Roy Cayetano (ed.), The People's Garifuna Dictionary (Belice: National Garifuna Council, 1993). De Cayetano tambi[en pueden consultarse: Lemesi lima Uremu Garifuna (Belmopan: Government Printers, 2002); y Towards a common Garifuna orthography (Belice: National Garifuna Council, 1992).

25 Cayetano, 171.

26 Cada uno de esos grados implica una función, razón por la que aparecerá glosado según el caso (como fut, rel Oipfv).
} 
verdadero sufijo -ña: $n$-arihu- $\tilde{a} a-i$ 'lo estoy viendo'. Esta forma morfologizada coexiste con usos que se asemejan más a un auxiliar en los que funciona como cópula (11), o en saludos ¿Ida b-iña? ‘Cómo estás?', Prisila t-iña 'Es Priscila'.

\section{(11) M-aberesagu-ti-na \\ üdüroü rügü-ña-i-la \\ neG-importar-aor-1sG \\ pescado rSt-proG-3SG.m-cmp \\ l-ichiga n-un porke n-uma-ña-i \\ muna-da. \\ 3sG.m-dar 1sG-dat porque 1sG-con-proG-3sG.m casa-en. \\ 'No me importa que solo me viva dando pescado, porque está conmigo en casa'}

El tercer auxiliar del garífuna de Colón es uma, que funciona prácticamente de la manera descrita para las variedades beliceñas por parte de Taylor y Devonish y Castillo, y que se ilustra en (12), para expresar el presente perfecto en lo que aquí se analiza como construcción objetiva, aquella en la que ambos participantes se indexan en el auxiliar y casi siempre con manifestación léxica del paciente. Como se aprecia en (12), uma se reduce $\mathrm{a}-a$; esto ocurre con las tres personas del singular.
Dara
$n-a-l i$
bena
Abrir 1sG-pfc-3sG.m
puerta

'He abierto la puerta'

Para situaciones transitivas cuyos objetos son indefinidos, el auxiliar toma la forma epentetizada $h a$ - en el caso de las formas $\operatorname{cortas}^{27}$. En el caso de los verbos que no tienen formas cortas la

27 Con respecto a esta forma, Devonish y Castillo señalan lo siguiente: «In the case of the perfect suffix, $-(h) a$, Taylor (51) treats $-h a-$ as the basic form, with the /h/ deleted when it occurs after a stem final $/ \mathrm{a} /$. Because of our suspicion that the perfect suffix, $-(h) a-$, is related to the perfect auxiliary forms $-u m a-\sim-a-$, we prefer for now to treat $-a-$ as the base form of the suffix with $/ \mathrm{h} /$ [sic] inserted when preceded by a vowel other than /a/. Further evidence will resolve this issue». Este autor se inclina por la propuesta de Devonish y Castillo por considerarla más plausible. La propuesta de Taylor no da cuenta de casos en que el perfecto de formas cortas terminadas en /a/ mantiene la [h] epentética, como en dara hadina bena 'he abierto la puerta'. 
presencia del auxiliar es prácticamente imperceptible, probablemente porque se funde con la vocal de la raíz. En (13), se comparan las formas perfectivas de un verbo intransitivo achülura 'llegar' en su forma corta chülï, uno transitivo adahira 'guindar' con forma corta dahi, y uno transitivo ariha 'ver', sin forma corta.

$\begin{array}{llll}\text { (13) } 1 \text { 1. chülü hadina } & \text { dahi } & \text { hadina } & \text { ariha-dina } \\ \text { 2. chülü hadibu } & \text { dahi } & \text { hadibu } & \text { ariha-dibu } \\ \text { 3. chülü hali/haru } & \text { dahi } & \text { hali/haru } & \text { ariha-li/-ru } \\ \text { 1. chülï hadiwa } & \text { dahi } & \text { hadiwa } & \text { ariha-diwa } \\ \text { 2. chülü hadiü } & \text { dahi } & \text { hadiü } & \text { ariha-diü } \\ \text { 3. chülü hadiña } & \text { dahi } & \text { hadiña } & \text { ariha-diña }\end{array}$

Un uso de este auxiliar, no señalado por los autores mencionados, es para expresar modalidad imperativa (exhortativa), como en (14), tomado de la contraportada de la Gramática escolar garífuna ${ }^{28}$.
(14) M-afedira
$w a-m a-i$
wa-ñeñe
neG-perder
1pl-imp-3sG.m
1pl-lengua
'No perdamos nuestra lengua'

El cuarto auxiliar, umut, funciona de manera similar a uma, en tanto que se utiliza exclusivamente para la construcción objetiva en el aoristo. Devonish y Castillo señalan que este auxiliar expresa aspecto puntual; en realidad, la puntualidad es una de sus lecturas, como ocurre con el aoristo, pero no es la única; en (15) difícilmente se le puede asignar una lectura puntual a esa oración, la cual se refiere a lo que hacía una persona recién fallecida. La narradora está hablando de un hecho que había acaecido dos días antes. Por lo tanto, es lícito concluir que la función principal de ese auxiliar es la de portar los afijos de agente y paciente.

28 Secretaría de Educación, Garüdia lánina lafansehaun hererun garinagu. Gramática escolar garífuna (Tegucigalpa, 2002). 


\section{(15) Sun le gabaral-umut-i l-adüg-un lun}

Todo dem.m poder3sG.m-aor.o-3sG.m 3sG.m-hacer-nom para $l$ abagarid-un adïga l-umut-i

3sG.m-vivir-nom hacer 3sG.m-aor.o-3sG.m

'Todo lo que podía sembrar o hacer para sobrevivir, lo hacía'

De los otros tres auxiliares que Taylor incluye, ani, iabu, y hamuga, el primero no se analizará como tal pues en la variedad estudiada se comporta como un nominalizador para formas subordinadas, como en (16). Al igual que en el caso del futuro, en la variedad estudiada, el paso de la morfologización se completó en esta forma, como se evidencia el paso del prefijo de persona del supuesto auxiliar al verbo principal; es decir de un posible ${ }_{\mathrm{VP}}\left[_{\mathrm{V}}[\text { ariha }]_{\mathrm{V} \text { Aux }}[l-\right.$ ani-na $\left.]_{\mathrm{Aux}}\right]_{\mathrm{VP}}$ se pasó $\mathrm{a}_{\mathrm{v}}[l-\text { arihi-ni-na }]_{\mathrm{V}}$.

\section{(16) Ligia h-anügu-ba-i lun me-ti bugaha}

3SG.m 3pl-traer-rel-m para fut-enf pdo

l-arihi-ni-na

3sG.m-ver-nom-1sG

'A él fue que trajeron para me viera'

En cuanto a $i a b u$, en el proceso de documentación esta forma no se registró; hay una forma similar iabi, que significa 'venir', pero que se utiliza como un simple verbo de movimiento, como se ilustra en (17a-b).
(17a) Ligia l-iabi-n
ma-digi-t-i
3sG.m 3sG.m-venir-nom neG-sacar-aor-3sG.m
'Vino sin haber pescado nada'
b) Ligia l-iabi-ña

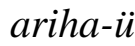
3sG.m 3sG.m-venir-proG ver-2pl
Viene a verlos' ${ }^{29}$
(Adaptado de Suazo 1991: 103)

29 Adaptado de Suazo, 148. 
En lo que respecta a hamuga, se ha recopilado principalmente como un marcador de modalidad condicional. Suazo presenta el paradigma de lo que el autor llama «caso optativo», en el que hamuga evidentemente funciona como un auxiliar, en este caso de modalidad volitiva; esta función de hamuga se ilustra en (18a), intransitiva, y (18b), transitiva ${ }^{30}$.
(18a) Hila
b-amuga
l-uagu
sun uriba-ti
Morir 2sG-vol
3sG.m-por
todo malo-m
le
b-adügu-ba-i
$n-u n$
dem.m 2sG-hacer-rel-m
$1 \mathrm{SG}-\mathrm{dat}$
'Ojalá te murás por todo lo malo que me hiciste'
(18b) Ariha n-amuga-bu
Ver $1 \mathrm{sG}-\mathrm{vol}-2 \mathrm{sg}$
'Ojalá me vieras'

La descripción precedente permite concluir que los auxiliares del garífuna de Colón son cinco: (u)ba, (i)ña, (um)a, umut, hamuga. Los primeros dos exhiben un altísimo grado de gramaticalización que les ha hecho perder su autonomía sintáctica y en sus usos principales son ya prácticamente sufijos; eso sí, coexisten con versiones menos gramaticalizadas que revelan su estatus como auxiliar. En un proceso similar, pero no tan avanzado se encuentra el auxiliar de perfecto, (um) $a$, el cual mantiene su autonomía en la construcción objetiva, pero en las construcciones intransitivas y transitivas de objeto indefinido de verbos sin forma corta muestra un elevado nivel de erosión fonológica. El auxiliar de aoristo para construcción objetiva, umut, es el único que actualmente se comporta como un auxiliar en el sentido estricto, esto es, como forma libre portadora de morfemas gramaticales. En cuanto a hamuga, su función y uso más frecuente es la de marcador de modalidad condicional, para la cual esprácticamente una forma invariable.

30 Suazo, 148. 


\section{Categorías verbales}

Las categorías gramaticales que son relevantes para la descripción del verbo garífuna son persona - categoría que se manifiesta tanto en verboides como en formas plenas-, aspecto, voz (pasiva), modo y tiempo; esta última expresada mediante formas libres.

\section{Persona}

La categoría de persona se expresa mediante afijos; los prefijos expresan sujetos ( $n$-ariha 1 sG-ver 'voy a ver'), mientras que los sufijos indexan sujetos en ausencia de prefijos (ariha-ti-na veraor-1SG 'veo'), y objetos cuando la forma verbal contiene un prefijo (b-ariha-na 2sG-ver-1sG 'me ves') ${ }^{31}$. Una excepción la constituyen los sufijos de construcciones de propósito, formadas con verbos de movimiento, como en (17b), arriba, donde solo aparece una marca de persona, la cual refiere al objeto. Los morfemas ligados de persona del garífuna son prefijos, infijos y sufijos, cuya forma gira en torno al tema consonántico de cada persona; en términos generales, lostemas son nasal alveolar para primera [n], bilabial sonoraparasegunda [b], alveolar (lateral, nasal, vibrante, oclusiva [1, n, R, t]) para tercera; para el plural los temas son deslizante bilabial para primera plural $[\mathrm{w}], \mathrm{y}$ aspirada $[\mathrm{h}]$ para segunda y tercera (en estas últimas, los sufijos tienden a ser-iïy-ña, respectivamente). El paradigmade infijos aparece en (19); se utiliza, entre otros, en el verbo estativo $a .$. $h a$; así pues $a-n a-h a$ 'estoy', $a-b a-h a$ 'estás', y así sucesivamente.

(19) Infijos pronominales del garifuna

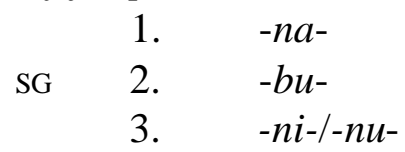

31 Los verbos intransitivos con pronombre sufijado son agramaticales (*arumuga-na 'duermo') y cuando se puede añadir el sufijo de persona causan una lectura transitiva; así pues, $t$-awiwanda-na (< awiwnada 'vivir') significa 'me utiliza', t-awiwandu-ña-dibu 'se está aprovechando de vos'. 


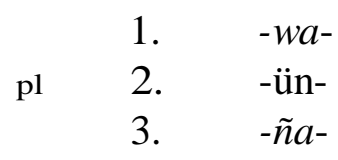

El siguiente paradigma de afijos pronominales lo constituyen los prefijos; éstos se utilizan en función de sujeto en construcciones simples y compuestas. En las simples se utilizan para expresar los aspectos inmediato, progresivo, el futuro simple, la voz pasiva y formas subordinadas. En las formas compuestas, los prefijos se utilizan en el auxiliar. El paradigma de prefijos se presenta en (20).

(20) Prefijos pronominales del garífuna

$\begin{array}{lll} & 1 . & n- \\ \text { SG } & 2 . & b- \\ & 3 . & l-/ t- \\ & \text { 1. } & w a- \\ \text { pl } \quad 2 . & h- \\ & \text { 3. } & h a-\end{array}$

Los sufijos pronominales se utilizan en los siguientes seis paradigmas, aunque en realidad la forma es la misma, con excepción del perfecto y el resultativo, variando solamente el auxiliar en el caso de las formas compuestas, o el sufijo de aspecto en el caso de las simples.

(21a) Sufijos pronominales, objeto (verboides)

$\begin{array}{lll} & 1 . & -n a \\ \mathrm{SG} & 2 . & -b u \\ & 3 . & -i \sim-l i /-u \sim-r u \\ & 1 . & -w a \\ \mathrm{k} 1 & 2 . & -\ddot{u} \\ & 3 . & -\tilde{n} a\end{array}$


(21b) Sufijos pronominales, sujeto en modo completivo

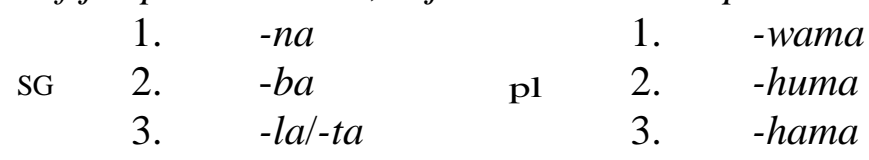

\section{Aspecto}

A diferencia de la persona gramatical, que se expresa tanto en verboides como en formas plenas, el aspecto solo se expresa en las segundas; de hecho, es la presencia de esta categoría la que les da el estatus de plenas a las formas verbales.

\section{Figura 1. Aspecto en garífuna}

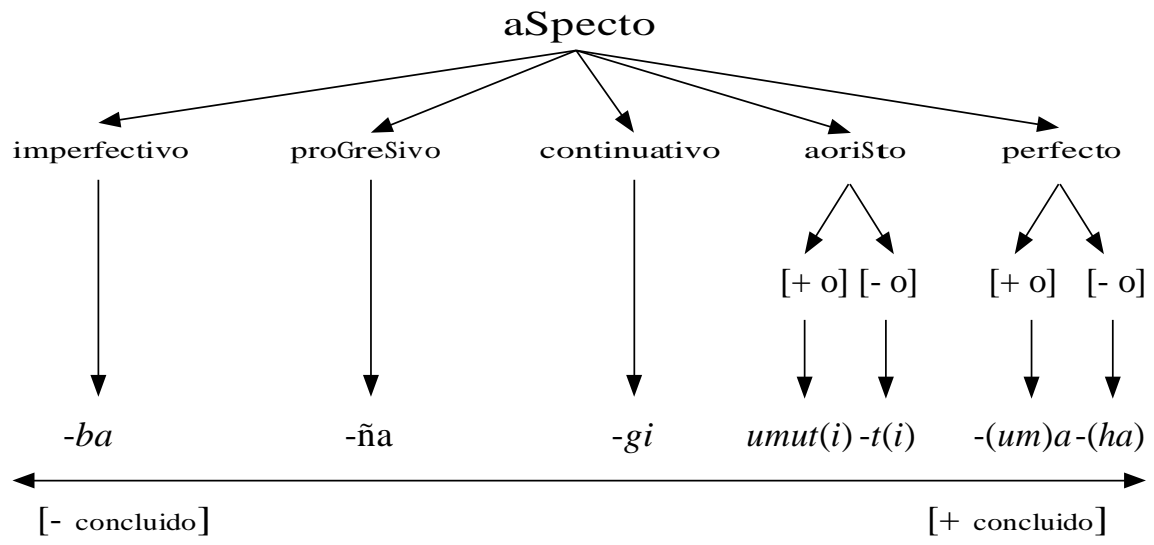

La figura 1 muestra las cinco subcategorías aspectuales del garífuna; de esas, dos -el aoristo y el perfecto- distinguen entre construcción objetiva y no objetiva. La primera es aquella que indexa a ambos participantes con o sin realización léxica del objeto, mientras que la no objetiva es la que no indexa al objeto aun cuando éste esté realizado léxicamente, como se aprecia en el par de (22). El objeto en la construcción objetiva tiende a ser siempre definido e individuado. Las cinco distinciones aspectuales del garífuna se pueden ordenar en 
un continuum de menor a mayor grado de compleción de la situación verbal, siendo el imperfectivo y el perfecto los dos extremos.

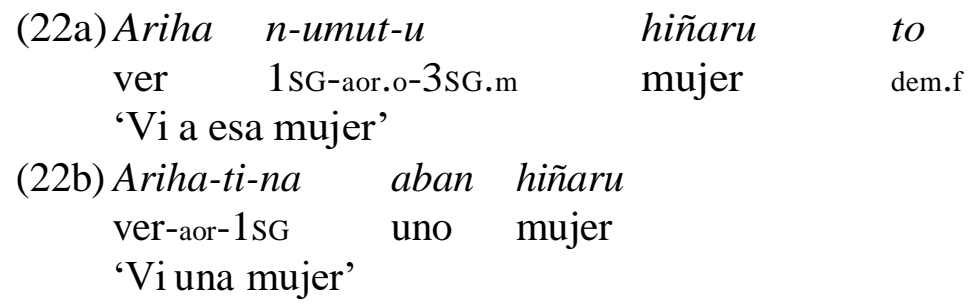

El aspecto imperfectivo se expresa en contextos específicos, y no tiene una frecuencia como la tienen los aspectos imperfectivos en otras lenguas, como el teribe (chibcha, Panamá) donde incluso son el miembro no marcado de una oposición [ \pm perfectivo]. Hay que tener presente que en garífuna, la frecuencia de verboides en oraciones subordinadas, así como el uso del aoristo en oraciones principales, lo mismo que la tendencia a construcciones nominales para la codificación de funciones como la posesión ${ }^{32}$, hacen que ciertas formas aspectuales, como es el caso del sufijo $-b a$ cuando expresa aspecto imperfectivo, tengan contextos morfosintácticos específicos, y con ello su frecuencia se ve reducida. El uso de - $b a$ en tanto marcador de aspecto imperfectivo se reduce a oraciones que expresan el fondo o antecedente de una situación (background sentences) sobre la que un hecho puntual va a ocurrir, como en (23); es decir, para el conocido «esquema incidental $»^{33}$. Que se trata de un imperfectivo se confirma al contrastar prácticamente el mismo estado de cosas ya consumado, $y$ codificado como una frase verboidal, como en (24).

32 Por ejemplo en (i):

(i) Ibidie-t-i n-un atiri-la irumu l-au Desconocer-aor-3SG.m 1sG-dat cuánto-cmp año 3sG-con 'No sé cuántos años tiene él [lit. cuántos años con él]'

33 Comrie Bernard, Aspect: An Introduction to the Study of Verbal Aspect and Related Problems (Cambridge: Cambridge University Press, 1976). 
(23)Dan le n-agañeiha-ba-nu gaderu tubalaba, Cuando 1sG-comprar-ipfv-pl cuatro rueda, ragu ha-muti-na sudara agarrar 3pl-aor.o-1sG policía 'Cuando compraba el carro, me agarró la policía' (24) Larigiñe n-agañeiha-nu gaderu tubalaba, Después 1sG-comprar-pl cuatro rueda, ariha ha-muti-na sudara ver 3pl-aor.o-1sG policía

'Después de comprar/de que compré el carro, me vio la policía'

Otros usos de $-b a$, como en las construcciones de foco, ya no corresponden a su función de imperfectivo, sino que corresponden a una función de marcador de relativo, de futuro y hasta de imperativo (ver la siguiente sección), las cuales, como se explicó anteriormente representan diversos estadios de gramaticalización del otrora marcador de modo irrealis.

El aspecto progresivo se expresa mediante el sufijo - ña, en formas que constan de un prefijo de agente y un sufijo de paciente, en el caso de las construcciones transitivas, y un prefijo de sujeto en las intransitivas, tal y como se ilustra en (25a-b). Además de expresar situaciones en desarrollo, la versión menos gramaticalizada del progresivo se utiliza como cópula, tal y como se explica en el apartado anterior.
(25a) $N$-adararu-ña-i
bena
1SG-abrir-proG-3SG.m puerta
'Estoy abriendo la puerta'

\section{(25b)}

$\begin{array}{ll}\text { L-arumugu-ña } & \text { Beto } \\ \text { 3sG.m-dormir-proG } & \text { Beto }\end{array}$


En cuanto al aspecto continuativo, expresado mediante el sufijo $-g i$ y analizado por Taylor $^{34}$ como un marcador de aspecto durativo, éste difiere del progresivo en que este último presenta la situación en su desarrollo, mientras que el primero expresa que aún ocurre, aunque no necesariamente de manera dinámica, como se aprecia en (26a); al negarse el aspecto continuativo se convierte en acción inconclusa, o aspecto incompletivo (26b).

\section{(26a) Sandi-gi-li \\ Enfermo-cnt-3sG.m \\ 'Continúa enfermo' \\ (26b) $M$-iabi-gi-dibu \\ neG-venir-cnt-2sG \\ 'Todavía no has venido'}

El aoristo en garífuna expresa una situación indefinida en tiempo pero con un modo de acción ingresivo, lo que explica que se tienda a traducir como pasado y puntual; no obstante, no siempre se le puede asignar una lectura pasada, como en (27). Esa característica del aoristo es la que permite que ese mismo morfema sea el que se utilice tanto para la predicación adjetiva (28), como para expresar énfasis (29); los dos rasgos semánticos del aoristo, la atemporalidad y la puntualidad, son esenciales e inherentes a esas funciones, respectivamente.

\section{(27)Busien-ti-na na-koturu-ni-bu}

Querer-aor-1sG 1sG-copular-nom-2sG

'Quiero tener relaciones sexuales con vos' 
(28) Luagu le n-aruhudaha-ba b-un keiti proudu-ti-na

Por eso, $1 \mathrm{sG}-\mathrm{enseñar-fut} 2 \mathrm{sG}-\mathrm{dat}$ como orgulloso-aor-1sG

l-un h-ererun n-aguburi-gu

3sG.m-dat 3pl-lengua 1sG-padres-pl

'Por eso te voy a enseñar, porque estoy orgulloso de la lengua de mis padres'

(29) Nein-ti l-agúmucha úraga le

Ahí-enf 3sG.m-terminar historia def.m

'ahí termina la historia'

La construcción objetiva en el aoristo es la que invita más a una lectura puntual precisamente porque, al indexarse ambos participantes, la situación tiene un punto de inicio - el agente- y un punto de llegada — el paciente - y de ahí la impresión de que expresa puntualidad, como en (30); sin embargo, como se advirtió en (15), no siempre es posible asignarle una lectura puntual a la construcción objetiva en el aoristo.

\section{(30)Buda ha-mut-i garawon \\ Recoger 3pl-aor.o-3sG.m tambor \\ 'Ellos recogieron los tambores'}

La última distinción aspectual del garífuna, el perfecto, es la única que expresa un evento en su estado concluido (31). Dentro de la perfectividad, en tanto compleción de un estado de cosas, el perfecto expresa principalmente anterioridad (32) y estado resultante (33) ${ }^{35}$ en el primer caso, los informantes sistemáticamente traducen el perfecto al español como 'ya + pretérito'.

35 Así pues, mientras chülüti significa 'llega' o 'llegó', chülühali significa 'llegó y se quedó'. 
(31) Tati-ha-dina

Empezar-pfc-1sG

'Ya empecé a lavar'

(32) Sun-n-a-li

Todo-1sG-pfc-3sG.m achibagua

lavar

n-wadagimari

1sG-trabajo

'He terminado todo mi trabajo'

\section{(33)Lidan to mabei-ha-li ligie meb-aranser-un-i}

Cuando dem.f secar-pfc-3sG.m cuando fut 2 sG-preparar-nom-m

b-ani almasigu

2sG.pos almácigo

'Cuando esté seco [cuando se haya secado], entonces preparás tu almácigo’

\section{Modo}

El modo y las diversas modalidades se expresan en garífuna mediante a) morfología ligada, b) auxiliares, c) operadores modales que toman morfología flexiva, y d) operadores modales invariables; los dos últimos toman como su objeto a un verboide. La morfología flexiva se utiliza para expresar el modo imperativo y la modalidad epistémica, principalmente. Para expresar el imperativo se adhiere otra forma gramaticalizada del auxiliar $u b a$, es decir, el ya sufijo - $b a$, a las formas cortas de los verbos que las tienen. Así pues, la forma imperativa de eiga 'comer', ásaragua 'rasurarse', ariha 'ver' y agamba 'escuchar' serán howba, saguaba, arihaba y agambaba, respectivamente. A las formas imperativas se les puede adherir un sufijo de objeto $-i$, masculino, $-u(n)$, femenino, y $-\tilde{n} a$, plural. De este modo ariha-baun, agamba-ba-i y ariha-ba-ña significan 'véala', 'óigalo' y 'véalos', respectivamente. Como ha quedado dicho, cuando el destinatario es plural se utiliza el auxiliar uma, el cual toma la forma huma para segunda persona plural y wama para primera persona plural, en el caso de modalidad exhortativa. De este modo las correspondientes formas plurales de un verbo como eiga 'comer' serán how huma ('coman') y how wama 'comamos'; del mismo modo, las de un verbo intransitivo 
como arumuga 'dormir' serán arumuga huma ('duerman') y arumuga wama ('durmamos'). En el caso de verbos reflexivos se adhiere el sufijo-gua antes del sufijo imperativo, aún en presencia del pronombre reflexivo ongua; la forma correspondiente a 'veámonos' entonces será Ariha-gua wa-ma ongwa (ver-med $1 \mathrm{pl}$-imp refl) y una construcción como 'véase en el espejo' será Ariha-gua-ba b-unguo t-idan isubusei (ver-mid-imp 2sG-ref1 3sG.f-en espejo). Como en muchas lenguas, existen formas supletivas imperativas en ciertos tipos de verbos ( $p$. ej. de movimiento); en garífuna, algunas de esas son beiba 'vaya' y keymoun 'vamos' de ñüdü 'ir'; o higabu 'venga' de numbin 'venir';

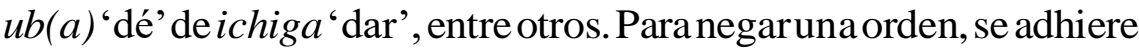
el prefijom-; asípuesde ayahuaha 'llorar' se obtiene ayahuaba 'llore' y m-ayahuha-ba 'nollore'.

Otra modalidad expresada por medio de morfología ligada es la epistémica; esta se expresa de manera discontinua, prefijando el segmento $g(a)$-, que Taylor ${ }^{36}$ denomina atributivo, más el sufijo-ei, que es isomorfo con el sufijo de gerundio. Esa construcción se utiliza para expresar habilidad en la mayoría de los casos, un ejemplo del cual es (34), y en ocasiones expresa acciones habituales, como en (35). Cuando se desea expresar modalidad epistémica en lo que equivale a una conjunción correlativa, el sufijo discontinuo -ei se sustituye por el sufijo - di en ambos correlatos de la construcción, la cual se completa mediante el uso del auxiliar progresivo iña, al cual se le prefija el pronombre de sujeto (36).
(34) G-afilih-ei-t-i
eyeri lira
epS-nadar-epS-aor-3sG.m hombre dem.m
'Ese hombre sabe/puede nadar'
(35) G-ebereh-ei-t-i
epS-ventosear-epS-aor-3sG.m
'Se tira muchos pedos [es común en él]'

36 Taylor, 45. 
(36) G-alira-di wa-ña l-au garifuna, g-alira-di eps-leer-eps 1pl-prog 3sG.m-con garífuna, eps-leer-eps wagía l-au chumagü $1 \mathrm{pl} \quad 3 \mathrm{sG}-\mathrm{m}-\mathrm{con}$ mestizo

'Podemos leer no solo en garífuna sino también en mestizo [español]'

La modalidad epistémica se expresa en los verboides como un sufijo - gu, como en (37).

\section{(37) lun l-adüra-gu-ni-wa \\ para 3sG.m-cortar-epS-nom- $1 \mathrm{pl}$ \\ 'para que él nos pueda cortar'}

Además de los auxiliares uma e iña, utilizados parcialmente - en tanto que solo se utilizan para el plural - en la expresión de las modalidades descritas anteriormente, hay que incluir a hamuga, ya mencionado en el apartado anterior e ilustrado en (38a), el cual expresa modalidad volitiva. Para el plural se utiliza una forma que insinúa la unión del auxiliar uma, con evidente función irrealis, con hamuga, como se aprecia en (38b), adaptado de Suazo (148).

(38a) Hila b-amuga l-uagu sun uriba-tile

Morir 2sG-vol 3sG.m-por todo malo-m dem.m

$b$-adügu-ba-i n-un

2sG-hacer-rel-m 1sG-dat

'Ojalá te murás por todo lo malo que me hiciste'

(38b) Ariha wa-ma-muga-bu

Ver 1 pl-imp-vol-2sG

'Ojalá te veamos'

En cuanto a modalidades expresadas mediante operadores modales que toman morfología flexiva se encuentran el volitivo 
abusenra 'querer, necesitar' y el 'potestativo' gayara $\sim$ giara, que difiere de la construcción epistémica sintética en que esa tiende a expresar habilidad, mientras que la analítica expresa, además, posibilidad y permisibilidad (40). Gayara difiere de abusenra también en que sintácticamente se comporta como un predicado alto que toma un complemento oracional, por lo que siempre aparece en tercera persona del aoristo y su complemento es un verboide, como en (39a), transitivo y (39b), intransitivo. Gayara posee una forma supletiva para expresar negación (41). Tanto la forma negativa como la afirmativa pueden aparecer en la construcción objetiva, como se aprecia en (42) y (43).
(39a) Gayara-t-i
h-adürüru-ni-na
Poder-aor-3sg.m 3pl-contar-nom-1sG
'Pueden cortarme' ['Es posible que me cuenten']
(39b) Gayara-t-i
n-arumug-un
Poder-aor-3sG.m 1sG-contar-nom
'Puedo dormir' [lit. 'Es posible mi dormir']

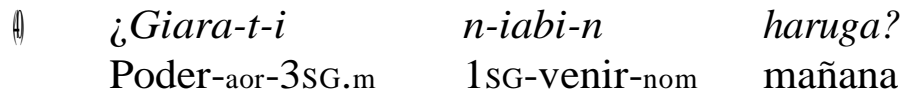
¿¿Puedo venir mañana?'
4) Sianti l-adumureiha
Poder-aor-3sG.m 3sG-hablar
'No puede hablar'
A Gayara l-umut-i adüragua
Poder 3sG.m-aor.o-3sG.m cortar

'Lo puede cortar'

A) Sian n-umut-i animalu le adina-güda

No poder 1sG-aor.o-3sG.m animal dem.m abordar-caus

'No pude subir al animal (albote)'

También es posible construir oraciones de modalidad potestativa codificando al paciente/sujeto como un sujeto dativo, como en (44). 
(4) Busien-ti-na yebu n-iabi-n ani

Querer-aor-1sG intentar 1sG-venir-nom pero sian-

$t-i \quad n-u n$

no poder-aor-3sG.m 1sG-dat

'Quería venir pero no puedo/pude'

En cuanto a abusenra, éste funciona como un verbo principal (45), así como un operador modal, función en la cual toma un verboide como su complemento (46). Abusenra posee una forma cuasisupletiva para la negación (47).

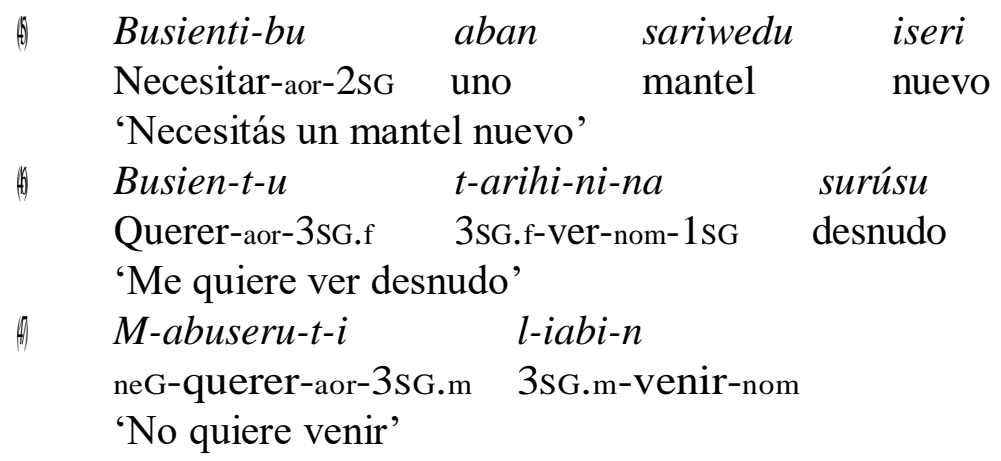

Existe, finalmente, en garífuna un operador modal invariable, mosu (< ingl. must), utilizado para expresar modalidad deóntica. Este operador toma verboides (48) como sus complementos. La única marca que mosu puede portar es la del complementizador -la en oraciones subordinadas (49).

Aun-ti b-abunag-un yuga, mosu b-adeir-un-u

Para-enf 2sG-sembrar-nom yuca, deber 2sG-encontrar-nom-3SG.f

geingücha furumiñe.

estaca de yuca primero.

'Para sembrar yuca primero tenés que encontrar estacas de yuca' 


\begin{tabular}{|c|c|c|c|}
\hline $\begin{array}{l}\text { Key-ti } \\
\text { Como-enf }\end{array}$ & $\begin{array}{l}\text { buga } \\
\text { pdo }\end{array}$ & $\begin{array}{l}\text { mosu-la } \\
\text { deber-cmp }\end{array}$ & $\begin{array}{l}\text { h-aluaha } \\
\text { 3pl-buscar }\end{array}$ \\
\hline
\end{tabular}

Voz

La voz pasiva es una importante categoría en la dimensión verbal del garífuna. Se expresa mediante el morfema-(o)wa. A diferencia del aspecto, la voz pasiva se expresa tanto en verboides (50) como en verbos plenos (51); en estos últimos, el morfema pasivo precede al de aspecto, mientras que en los primeros se separa por la presencia del nominalizador -ni, convirtiéndose así en un morfema discontinuo, ow...wa, como se aprecia en (52). La expresión discontinua también ocurre en la negación, tal y como se ilustra en el par de (53).

1) Lubaragiñe w-adügüraha, Antes de 1p1-bailar dugú, hísie-ti-ti $\quad n$-un gustar-aor-enf 1sG-dat 'Antes de bailar el dugü me arreglaron [lit. 'fui arreglada'] y me gustó' $\begin{array}{lccc}\text { Meisturu afaru-wa-t-i } & \text { l-uagu } & \text { mиa } \\ \text { Maestro matar-paS-aor-3sG.m } & \text { 3sG.m-por } & \text { tierra }\end{array}$ 'Un maestro fue asesinado por tierra'

Q Danle l-achülüru-ba-i ora lun Cuando 3sG.m-llegar-rel-m hora para w-ownah-ow-ni-wa wa-yabui-ba-i Yurumein-giñe, 1pl-mandar-paS-nom-pas 1pl-venir-rel-m San Vicente-desde, anura-rügü-ti-wa ñin-giñe t-idan üruwa guriara huir-rSt-aor-1pl allá-desde 3sG.f-en tres canoa 'Cuandollególahora en queíbamos a serenviados deSan Vicente, solo nos escapamos en tres barcos'

\section{(53a) M-arih-owa-t-u Maria}

neG-ver-paS-aor-3sG.f María

'María no fue vista' 
(53b)

$\begin{array}{lll}\text { Lun } & \text { m-arih-ow-ni-wa-ta } & \text { Maria } \\ \text { Para } & \text { neG-ver-paS-nom-paS-3sG.f } & \text { María }\end{array}$

'Para que María no fuese vista'

Una característica de la voz pasiva en garífuna es que no admite la expresión del agente; así pues en (54), proveniente de una canción propia de la comunidad de Cristales (Colón), el sintagma preposicional que sigue al sujeto pasivo no se refiere al agente; los hablantes son claros en decir que alguien no especificado en la oración leyó la carta; el sintagma preposicional se refiere a los protagonistas de la historia, no a quien leyó la carta.

\section{(54) $L i$-wa-t-u garada ha-uagu igeragu-tiñ}

Leer-paS-aor-3sG.f carta 3pl-por divorciado-m.pl 'La carta de los divorciados fue leída' [*'La carta fue leída por los divorciados']

La construcción pasiva se utiliza en construcciones impersonales, como en recetas (55).

(55) Lun t-adüga pulanbredi, aban t-elech-un Para 3sG.f-hacer pan de guineo, entonces 3sG.f-pelar-nom disi bimena diez guineos

funaru; u-wa ligia murusun kanela ñein-hin maduro; poner-pas entonces poco canela ahí-para 'Para hacer pan de guineo pela diez guineos maduros y se le pone un poco de canela'

\section{Tiempo}

El tiempo es la categoría menos relevante del verbo garífuna. Se expresa principalmente mediante partículas temporales, con 
excepción del futuro, el cual — dado el proceso de gramaticalización ya comentado sobre el auxiliar $u b a-$ se expresa mediante el sufijo - ba, como en (56). Alternativamente existe la partícula futura $m e$, la cual expresa una especie de prospectividad con respecto a los eventos (57). En cuanto a eventos pasados existen tres partículas, buga, bugaha y meha, que según Suazo ${ }^{37}$ expresan pasado reciente, la primera, y pasado remoto, las otras dos; en la práctica, sin embargo, la más frecuente es la primera y, como se aprecia en (58), se utiliza como partícula de pasado, simplemente; la situación narrada en (58) había ocurrido unos treinta años atrás ${ }^{38}$. Hay que hacer notar que el uso de estas partículas no es obligatorio; así pues, un evento que se narra puede haber ocurrido en retrospectiva y sin embargo as partículas no se utilizan, como en (59), que proviene de una historia sobre algo ocurrido mucho tiempo atrás.

(56) B-afaru-ba-dina

2sG-pegar-fut-1sG

'Me vas a pegar'

(57)Busien-t-i me buga binu; uwa-ti; sanudelu uwa-ti; Necesitar-aor-3sG.m fut pdo vino; neG-enf; candela neG-enf, u-ti-na aban korason de hesusu, aban sandu ñein, poner-aor-1sG uno corazón de jesús, uno santo ahí,

\section{lun me-ti buga giara-la l-adünama me lubarugume} para fut-enf pdo poder-cmp 3sG.m-lucir fut mientras tanto 'Iba a necesitar guaro, pero no había; candelas tampoco había; puse un Corazón de Jesús, o un santo ahí para poder lucir mientras se iba preparando el altar'

37 Salvador Suazo, Lila Garifuna. Diccionario Garífuna-Garífuna-Español (Tegucigalpa: Litografía López, 2011).

38 En la formación de preguntas sí se hace más evidente su función temporal, sobre todo porque al estar la posición inicial ocupada, la segunda posición la debe ocupar un verboide; así pues ¿Ida me liabin bumari? ‘¿Cuándo vendrá tu marido?’ se opone a ¿Ida buga liabin bumari? ‘¿Cuándo vino tu marido?’. 
(58) Nuguya buyei uguñe weyu ladüga-me buga aban weyu 1sG.f sahurín hoy día porque-fut pdo uno día l-iabi-n Manueli, le l-iroü finou Nadidi 3sG.m-venir-nom Manuel, def.m 3sG.m-hijo finado Nadidi arañaba n-un lun lan n-idi-n beyebu agúrabaha-i decir 1sG-dat para que 1sG-ir-nom playa esperar-3sG.m ladüga buga l-idiba-la óuchaha porque pdo 3sG.m-ir-cmp pescar 'Hoy día soy sahurín porque un día vino Manuel, hijo del finado Nadidi, a decirme que fuera a la playa a esperarlo porque iba a pescar'

(59) Luagu aban weyu n-udi-n ouchaha. Aban En uno día 1sG-ir-nom pescar. Entonces

n-adigi-n aban ovembun inegu; key ñüraü-hei 1SG-pescar-nom uno grandísimo mero; como pequeño-Ger

t-ubei yagana, sian n-umut-i adina-güda 3sG.f-ser canoa, no poder 1sG-aor.o-3sG.m montar-caus 'Un día fui a pescar y pesqué un mero grandísimo mero; como la canoa era pequeña no lo pude encaramar'

\section{Conclusión}

Se han esbozado las principales características morfológicas del verbo garífuna. Esta descripción, en especial lo referente al sistema aspectual, los auxiliares, las formas cortas y la indexación de participantes, es primer paso para continuar con el estudio de la sintaxis del verbo en esta lengua. Se ha identificado el sistema aspectual y representarlo de una manera coherente; asimismo, el haber 
identificado la construcción objetiva, cuyo desconocimiento llevó en algunos casos a ignorarla o a caracterizarla como un aspecto puntual, permite ahondar con propiedad en la sintaxis de esta lengua en el marco del proyecto de elaboración de la gramática descriptiva. Por ello, esta descripción no solo ordena muchos «cabos sueltos» de los estudios existentes, sino que permitirá avanzar en la urgente tarea de describir la estructura de una lengua amerindia cuya supervivencia en este nuevo milenio es incierta.

\section{Abreviaturas}

\begin{tabular}{ll} 
1,2,3 & persona gramatical \\
akt & aktionsart \\
aor & aoristo \\
aor.o & construcción objetiva en aoristo \\
cauS & causativo \\
cmp & complementizador \\
cnt & aspecto continuativo \\
dat & dativo \\
dem & demostrativo \\
enf & énfasis \\
epS & modalidad epistémica \\
f & femenino \\
fut & futuro \\
Ger & gerundio \\
imp & imperativo \\
ipfv & imperfectivo \\
m & masculino \\
mid & diátesis media \\
neG & negación \\
nom & nominalizador \\
obj & objeto \\
pas & pasivo \\
\hline
\end{tabular}




$\begin{array}{ll}\text { pdo } & \text { pasado } \\ \text { pfc } & \text { perfecto } \\ \text { pl } & \text { plural } \\ \text { pfc } & \text { perfecto } \\ \text { poS } & \text { posesión } \\ \text { proG } & \text { progresivo } \\ \text { rel } & \text { relativizador } \\ \text { rSt } & \text { restrictivo } \\ \text { SG } & \text { singular } \\ \text { Suj } & \text { sujeto } \\ \text { tam } & \text { tiempo, aspecto, modo } \\ \text { v } & \text { verbo } \\ \text { vol } & \text { modalidad volitiva }\end{array}$

